\title{
Adipose Tissue-Derived Stem Cell Therapy for Cavernous Nerve Injury-Induced Erectile Dysfunction in the Rat Model: A Systematic Review and Meta-Analysis Using Methodological Quality Assessment
}

\author{
Hyo Jung Park ${ }^{1,2}$, Hyunsuk Jeong, ${ }^{3, *}$, Yong Hyun Park ${ }^{1}$, Hyeon Woo Yim ${ }^{3}$, U-syn $\mathrm{Ha}^{1}$, \\ Sung-Hoo Hong ${ }^{1}$, Sae Woong Kim ${ }^{1}$, Na Jin Kim ${ }^{4}$, Ji Youl Lee ${ }^{1,2, *}$ \\ ${ }^{I}$ Department of Urology, College of Medicine, The Catholic University of Korea, Seoul, Korea \\ ${ }^{2}$ Department of Biomedicine \& Health Sciences, The Catholic University of Korea, Seoul, Korea \\ ${ }^{3}$ Department of Preventive Medicine, College of Medicine, The Catholic University of Korea, Seoul, Korea \\ ${ }^{4}$ Medical Library, The Catholic University of Korea, Seoul, Korea
}

Background and Objectives: Few studies were evaluated the effect of blindness on outcome in animal models, though a potential effect of blinding has been reported in clinical trials. We evaluated the effects of adipose tissue-derived stem cells (ADSCs) on cavernous nerve injury (CNI)-induced erectile dysfunction (ED) in the rat and examined how proper blinding of the outcome assessor affected treatment effect.

Methods and Results: We searched in Pubmed, EMBASE, Cochrane and Web of Science databases from inception to January 2019. We included CNI animal model, randomized controlled experiments, and ADSC intervention. Erectile function and structural changes were assessed by intracavernous pressure and mean arterial pressure (ICP/MAP) ratios, neuronal nitric oxide synthase (nNOS) levels, cavernous smooth muscle and collagen (CSM/collagen) ratios, and cyclic guanosine monophosphate (cGMP).

Results: Nineteen studies were included in the final meta-analysis. The ICP/MAP ratio of the ADSC treatment group increased compared to the control group ( $\left.\mathrm{SMD}=1.33,95 \% \mathrm{CI}: 1.11 \sim 1.56, I^{2}=72 \%\right)$. The nNOS level $(\mathrm{SMD}=2.29$, 95\%CI: $\left.1.74 \sim 2.84, I^{2}=75 \%\right), \mathrm{CSM} /$ collagen $\left(\mathrm{SMD}=2.57,95 \% \mathrm{CI}: 1.62 \sim 3.52 ; I^{2}=85 \%\right.$ ), and cGMP $(\mathrm{SMD}=2.96,95 \% \mathrm{CI}$ : $1.82 \sim 4.10, I^{2}=62 \%$ ) were also increased in the ADSC treatment group. Preplanned subgroup analysis was conducted to explore the source of heterogeneity. Five studies with blinded outcome assessment were significantly less effective than the unblinded studies (SMD $=1.33,95 \% \mathrm{CI}$ : $0.86 \sim 1.80$; $\mathrm{SMD}=1.81,95 \% \mathrm{CI}: 1.17 \sim 2.46$, respectively).

Conclusions: ADSCs might be effective in improving erectile function and structural change in CNI-induced ED. However, non-blinded outcome assessors might cause detection bias and overestimate treatment efficacy. Therefore, the ADSC efficacy must be further evaluated with a rigorous study design to avoid bias.

Keywords: Adipose tissue-derived stem cell, Erectile dysfunction, Meta-analysis

Received: November 29, 2018, Revised: February 9, 2019, Accepted: February 11, 2019, Published online: April 30, 2019

Correspondence to Ji Youl Lee

Department of Urology, The Catholic University of Korea, 222 Banpo-daero, Seocho-gu, Seoul 06591, Korea

Tel: +82-2-2258-6227, Fax: +82-2-2258-1536, E-mail: Uroljy@catholic.ac.kr

Co-Correspondence to Hyunsuk Jeong

Department of Preventive Medicine, The Catholic University of Korea, 222 Banpo-daero, Seocho-gu, Seoul 06591, Korea

Tel: +82-2-2258-7372, Fax: +82-2-532-3820, E-mail: Suejeong@catholic.ac.kr

${ }^{*}$ These authors contributed equally to this work.

(a) This is an open-access article distributed under the terms of the Creative Commons Attribution Non-Commercial License (http://creativecommons.org/licenses/by-nc/4.0/), which permits unrestricted non-commercial use, distribution, and reproduction in any medium, provided the original work is properly cited.

Copyright (c) 2019 by the Korean Society for Stem Cell Research 


\section{Introduction}

Prostate cancer is the second most frequent cancer and the fifth leading cause of cancer death in men worldwide (1). Approximately $80 \%$ of prostate cancer is identified as clinically localized and treated with radical prostatectomy (RP) (2). RP was the first treatment for localized prostate cancer that benefits overall survival and cancer-specific survival (3). Furthermore, the introduction of pioneering nerve-sparing robot-assisted laparoscopic radical prostatectomy has significantly improved the potency rate after RP (4). Despite surgical technical advancements, many patients experience erectile dysfunction (ED) after prostatectomy because of cavernous nerve injury (CNI) (5). Type-5 phosphodiesterase inhibitors (PDE5Is) are the first line drugs for ED, but PDE5Is provide only symptomatic relief of ED and do not offer a cure for the disease. Therefore there is a growing interest in developing therapies, including stem cell therapy, that offer a cure (6).

Stem cells are considered a potential therapy for restoring the injured cavernous nerve due to their paracrine effect on surrounding penile tissue and differentiation into smooth muscle, endothelium, and neuronal tissues (7). Mesenchymal stem cells (MSC) have capacity for self-renewal and differentiation into multiple lineages, including muscle, cartilage, bone, and fat (8). The paracrine effect leads to immunomodulation through the secretion of cytokines and growth factors that reduce inflammation and accelerate healing (9-11). The practical use of stem cells, such as embryonic stem cells and bone marrow derived-stem cells, is restricted due to ethical considerations, painful procedures, and the necessity of spinal anesthesia. Adipose tissue-derived stem cells (ADSCs) are a mesenchymal stem cell source that can be easily isolated from adipose tissue. ADSCs have abundant sources that are localized in subcutaneous adipose tissue throughout the body. Using minimally invasive liposuction, it is easier to obtain a large amount of ADSCs than other stem cells. In addition, ADSCs can be used for autologous or allogeneic transplantation in the body safely with less implant migration and foreign body reaction (12).

Up until now, two meta-analyses have been reported on the efficacy of stem cell therapy in the ED rat model (13, 14). Hou et al. (13) had analyzed the effect of ADSCs on ED induced by various causes, including diabetes mellitus, CNI, cigarette smoking, tunica albuginea, and radiation. Shan et al. (14) had shown that different sources of stem cell, such as neural embryonic stem cells, skeletal muscle derived stem cells, bone marrow derived stem cells, and ADSCs, recovered ED in CNI rat models. They had reported the efficacy of stem cell therapy in the ED rat model, but they did not evaluate the efficacy of stem cell therapy in consideration of methodological quality assessment. In clinical studies, randomization controlled trial (RCT) is a rigorous study design for evaluating intervention efficacy. Few studies were evaluated the effect of blindness on outcome in animal models, though a potential effect of blinding has been reported in clinical trials. Since the animal backgrounds were homogeneous, most of the studies did not mention the randomization procedure. It may not be necessary for animals to be blinded because they do not have treatment preference. However, blinding of the outcome assessor is important in animal studies because researchers may anticipate positive results. As in human RCTs, animal studies should also utilize high quality study design and analysis to ensure that the efficacy of intervention is not affected by conscious or unconscious bias (15). Therefore, we analyzed the effect of ADSCs on CNI-induced ED in rats. Moreover, we explored how proper blinding of the outcome assessor affected outcomes.

\section{Materials and Methods}

The review methodology was specified in advance and documented using the SYRCLE (Systematic Review Centre for Laboratory Animal Experimentation) systematic review protocol for animal intervention studies (Supplementary File S1). The review question was: what is the effect of ADSCs on ED in experimental CNI?

\section{Eligibility criteria}

The studies were included if they satisfied the following criteria: included a CNI animal model, was a randomized controlled experiment using ADSCs, and the article was written in English. Studies were excluded if they were a conference abstract or review article or had not determined the ICP/MAP ratio.

\section{Search methods}

We searched through PubMed, EMBASE, Cochrane and Web of Science databases for relevant studies published before January 28, 2019 using the following terms: "Cavernous nerve injury," "Prostatectomy," "Erectile dysfunction," and "Adipose tissue-derived stem cell." The full search procedure was documented in the Supplementary File S2. References were transferred to Endnote to eliminate duplicates. Two investigators ( $\mathrm{HJP}$ and $\mathrm{HJ}$ ) independently extracted the references by using the titles and abstracts as the first screening step. Then, full-text 
manuscripts were reviewed to select the final eligible studies. We also included animal studies to evaluate the effects of ADSCs with or without additional materials. Disagreements between the two investigators were discussed with a third reviewer (JYL) to reach a resolution.

\section{Data extraction}

Two investigators extracted study characteristics with a structured data extraction form. For each study, we extracted study characteristic data as bibliographic information that included the name of the first author, year of publication, rat species, rat age, origin of the cell, cell number, modification which was contained 2 or more combined treatments biologically, chemically or physically such as growth factor, drug or shock wave, the site of cell injection, and follow-up period. If studies evaluated multiple treatment groups in comparison to control groups, they were considered separate experiments. Our primary outcome was erectile function measured as an ICP/MAP ratio. Secondary outcomes were structural changes, such as neuronal nitric oxide synthase (nNOS) levels, the cavernous smooth muscle and collagen (CSM/collagen) ratio, and cyclic guanosine monophosphate (cGMP). All outcome data were extracted as the mean, standard deviation (SD), and the number of animals in both the intervention and control groups. If some studies expressed outcome data with the standard error (SE), we changed the data from $\mathrm{SE}$ to SD. When data were presented graphically, we extracted the numerical data from the graph using a WebPlotDigitizer version 3.8 (https://automeris.io/WebPlotDigitizer). If we could not find available outcome data from the journal, we attempted contacting the authors to inquire the raw data.

\section{Quality assessment}

Two investigators independently assessed the risk of bias for the studies based on the SYRCLE Risk of Bias tool for animal studies (16), which is derived from the Cochrane Collaboration tool for assessing risk of bias in randomized controlled trials. Each study was assessed for sequence generation, baseline characteristics, allocation concealment, random housing, performance blinding, random outcome assessment, blinding of outcome assessment, and incomplete outcome data that might influence the study outcome. Selective outcome reporting was not assessed because all of the studies did not report the use of a study protocol predefining primary and secondary outcomes. Judgements were expressed as "low risk of bias," "high risk of bias", or "unclear risk of bias." When assessing baseline characteristics, the studies were consid- ered low risk of bias if the rat species and age in both groups were described. If the method of randomization or allocation concealment was not described, we considered them as unclear. If random outcome assessment or blinding of outcome assessor were mentioned, we considered them as low risk of bias. Quality assessments were independently conducted by two investigators. Disagreements were resolved by discussion.

\section{Statistical analysis}

Outcome data were analyzed with Review Manager 5.3 (Cochrane collaboration). Differences between the intervention and control groups were expressed as standardized mean differences with $95 \%$ confidence intervals (CIs) for continuous variables. The heterogeneity was analyzed using $I^{2}$ statistics and defined as low (25\% to $\left.50 \%\right)$, moderate $(50 \% \sim 75 \%)$, or high (>75\%) (17). $I^{2}>50 \%$ indicated significant heterogeneity. The DerSimonian-Laird random-effect model was used when the $I^{2}$ value was $>50 \%$.

To explore the source of heterogeneity, we conducted preplanned subgroup analysis based on blinding of the outcome assessment (blinded, unblinded), origin of ADSC (autologous, allogenic, human), follow-up period $(<6$ weeks, $\geq 6$ weeks), route of administration (intracavernous, cavernous nerve, others) and co-intervention (yes, no). Publication bias was examined graphically with a contour enhanced funnel plot. If publication bias was suspected, we adjusted the estimate using the trim and fill method.

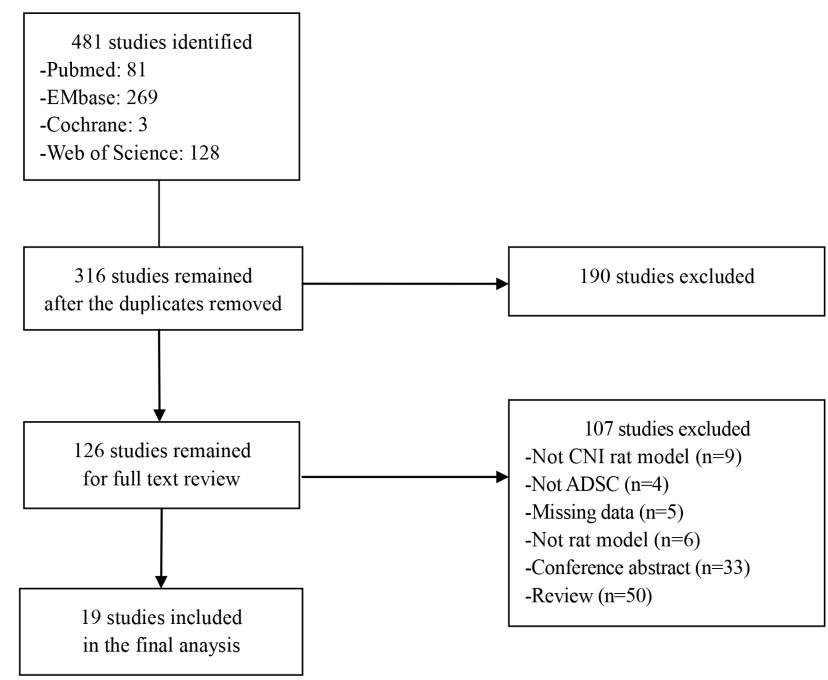

Fig. 1. Flow diagram of the studies included in the meta-analysis. 


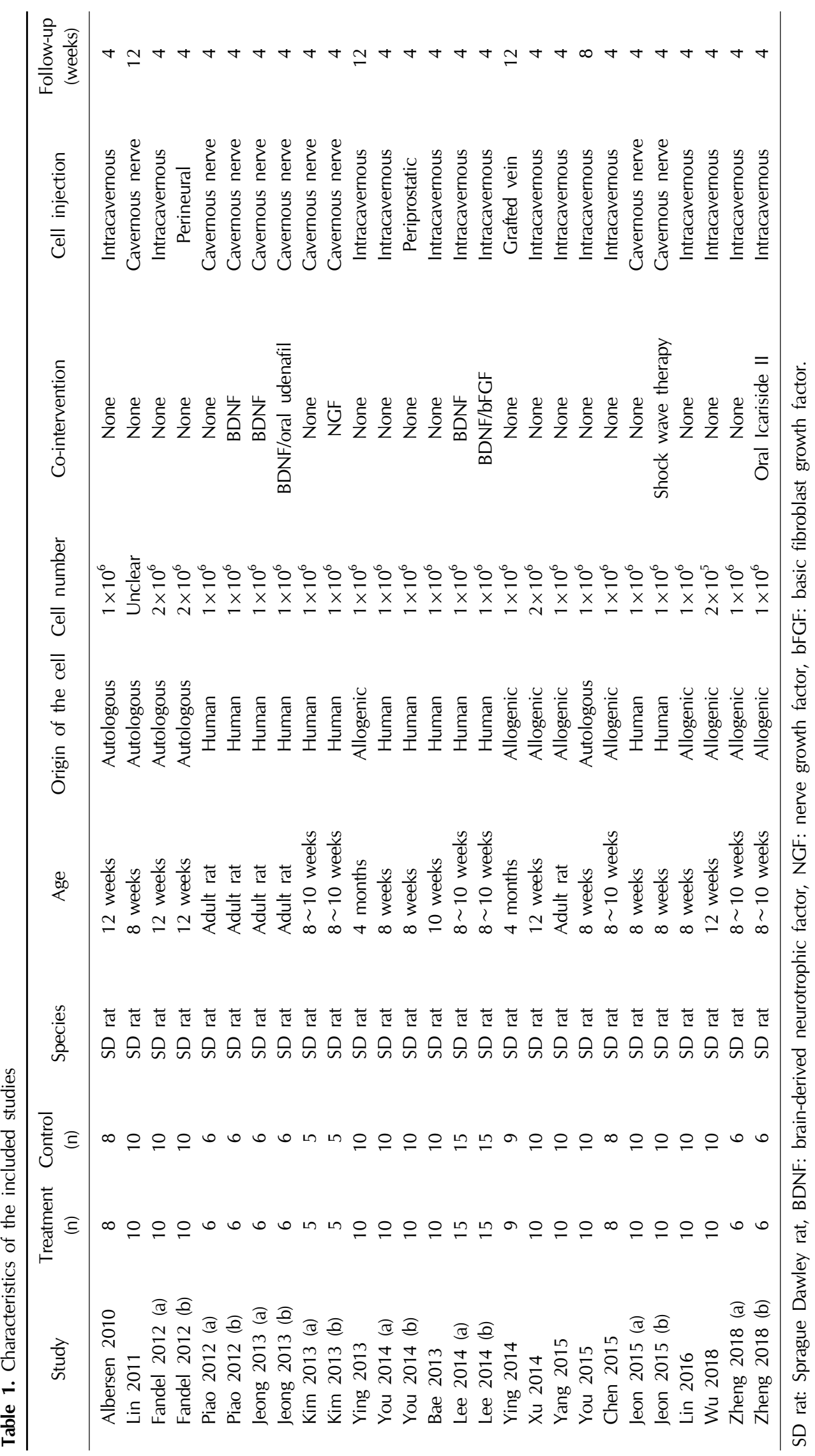




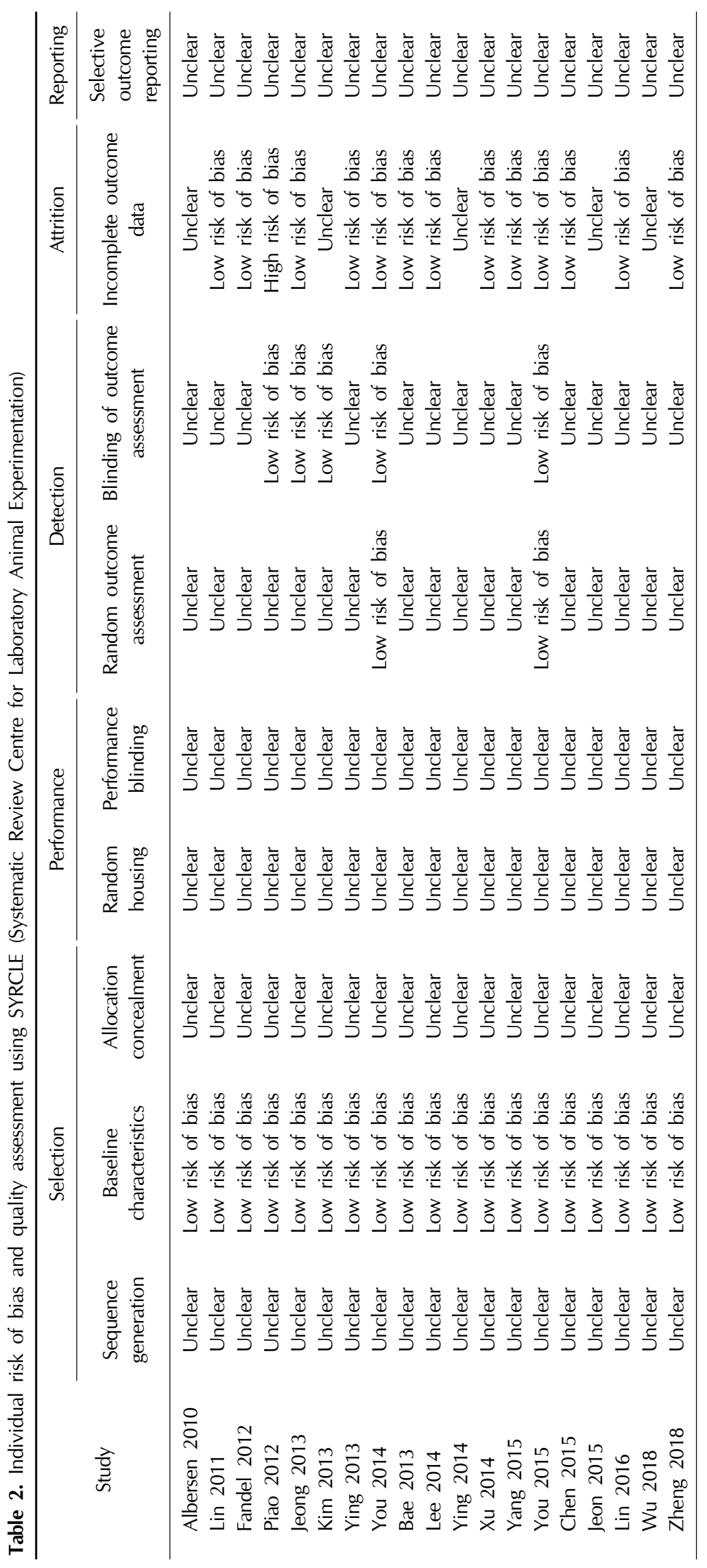




\section{Results}

\section{Search and study selection}

We identified 481 studies from PubMed $(\mathrm{n}=81)$, EMBASE $(n=269)$, Cochrane $(n=3)$ and Web of Science $(n=128)$ databases. After removing duplicate articles, 316 studies remained. We excluded 190 studies after a first screening on title and abstract. Another 107 studies were excluded after assessing the full text manuscript. Finally, 19 studies were included in this meta-analysis (Fig. 1).

\section{Study characteristics}

Characteristics of the 19 included studies are summarized in Table 1. Eight studies reported on multiple treatments and were considered separate experiments (18-25). A total of 27 animal experiments from 19 studies were included in this meta-analysis. Publication years were from 2010 to 2018. All of the studies used Sprague Dawley (SD) rats. Allogenic, autologous, and human ADSCs were administered in 8 (25-32), 4 (18, 33-35), and 7 studies, respectively $(19-24,36)$. Injected cell doses were $1 \times 10^{6}$ in 15 of the studies $(19-26,28-31,33,35,36)$. Two studies used $2 \times 10^{6}$ cells $(18,27)$. One study used $2 \times 10^{5}$ cells (32). Cell doses in one study were not clear (34). Six studies reported on multiple treatment groups that contained 2 or more combined biological, chemical, or physical treatments, such as growth factor, drug, or shock wave, respectively $(19-23,25)$. Thirteen studies injected ADSCs into the intracavernous (IC) route $(18,22,24-27,29-33,35$, 36). Five studies injected ADSCs into the injured cavernous nerves (19-21, 23, 34). One study injected ADSCs into the grafted vein (28). Two studies evaluated the effect of ADSCs between different delivery routes $(18,24)$. One of these studies compared injections into the IC route and the dorsal penile perineural space (18); the other study compared periprostatic implantation to IC injection (24). The follow-up periods were from 4 to 12 weeks.

\section{Risk of bias assessment in the included studies}

The results of study quality and the risk of bias assessment are shown in Table 2. All of the studies mentioned randomization but failed to provide details of the randomization procedure. As a result, the risk of bias in terms of sequence generation and allocation concealment was unclear. All of the studies reported that the rats were Sprague Dawley (SD) rats of similar age and weight at baseline. As a result, the risk of bias for baseline characteristics was low. Three studies mentioned similar housing conditions $(30,33,35)$ but did not report whether the rats were randomly housed during the experiment. As a result, the risk of bias related to random housing in the studies was unclear. All of the studies failed to report performance blinding. Five studies had mentioned that blinding of outcome assessment was applied during the experiment $(20,21,23,24,35)$. However, this was only related to the outcome assessment of structural change. Despite insufficient descriptions, we considered the risk of bias to be low since the study authors had attempted to maintain blinding of the outcome assessors. Two studies had reported that the outcome assessment was randomly conducted. Therefore, random outcome assessment was considered a low bias risk $(24,35)$. Thirteen studies did not have missing number of rats in each group to analyze. This item was considered low for risk of bias. In one study, the risk of attrition bias was assessed as high because there were discrepancies between the number of rats that completed the study and at baseline (23). In five studies, the number of rats per group was mentioned only once at baseline, and the number of rats completing the study was not reported. Thus, the risk of attrition bias was unclear $(19,21,28,32,33)$.

\section{Efficacy of ADSC on ED}

Eighteen out of the 19 studies had reported outcomes related to the ICP/MAP ratio. One study reported only the change in the ICP/MAP ratio from the baseline, and not the ICP/MAP ratio after treatment (18). Accordingly, we could not calculate the ICP/MAP ratio after the treatment from the reported data. We attempted to contact the corresponding author in order to obtain the raw data, but we received no response. Another co-author replied to email but he did not have the raw data. Thus, 18 studies were included in the quantitative analysis. As shown in Fig. 2, ADSC therapy significantly increased the ICP/MAP ratio (SMD 1.33, 95\% CI, 1.11 to 1.56 ) as compared to the control group. Moderate heterogeneity was observed $\left(I^{2}=72 \%\right)$. The nNOS level (SMD 2.29, 95\% CI 1.74 2.84; $I^{2}=75 \%$ ), CSM/collagen (SMD 2.57, 95\% CI 1.62 $3.52 ; I^{2}=85 \%$ ), and cGMP (SMD 2.96, 95\% CI 1.82 4.10; $\left.I^{2}=62 \%\right)$ were increased compared to the control groups. A random-effects model was used for the overall analysis.

\section{Subgroup analyses}

Preplanned subgroup analysis was conducted to explore the source of heterogeneity. When studies were compared between the blinding of outcome assessment, the studies with a blinding of the outcome assessment had shown a lesser effect on erectile function (1.33, 95\% CI: 0.86 to 1.80) than the studies that had not blinded the outcome assessment (1.81, 95\% CI: 1.17 to 2.46). Heterogeneity in 
A

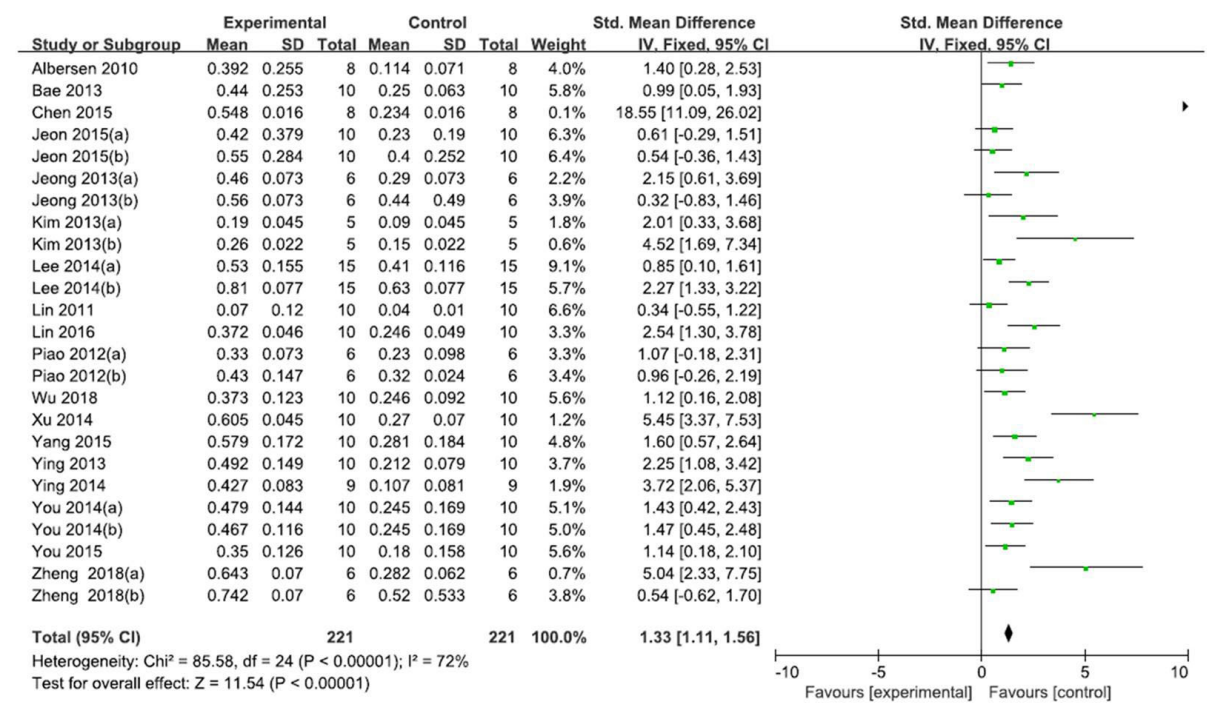

B

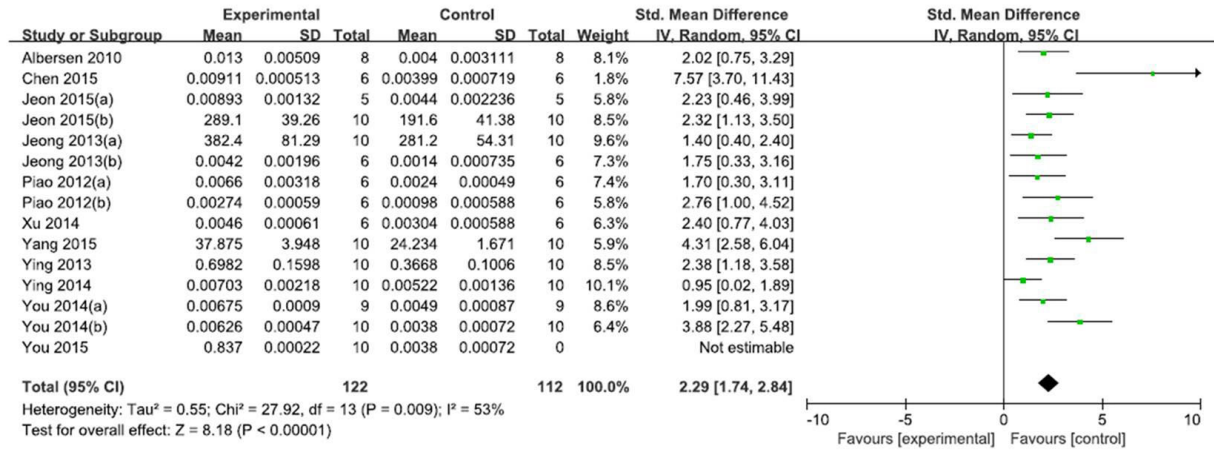

C

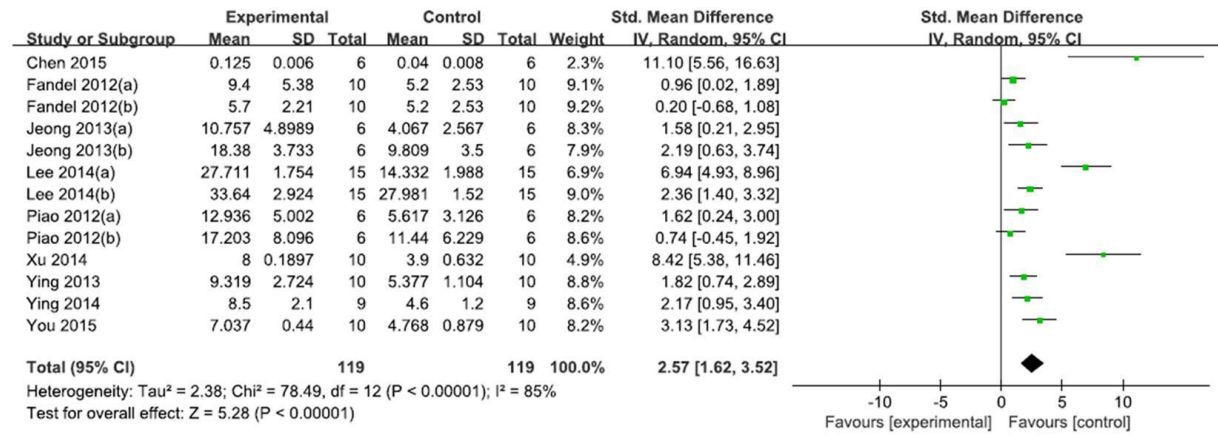

D

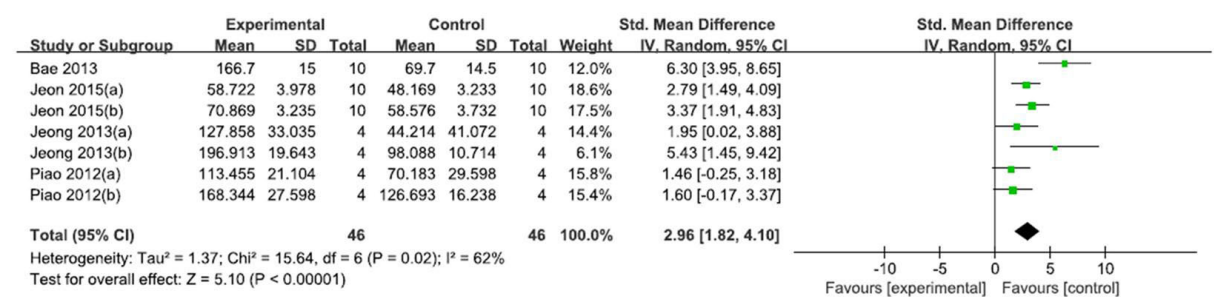

Fig. 2. ADSC effects on erectile function and structural change. (A) Intracavernous pressure and mean arterial pressure (ICP/MAP) ratio, (B) neuronal nitric oxide synthase (nNOS), (C) cavernous smooth muscle and collagen (CSM/collagen) ratio, and (D) cyclic guanosine monophosphate (cGCMP). 
studies with outcome assessment blinding reduced by minimal level $\left(I^{2}=23 \%\right)$. When studies were compared according to the origin of ADSC, allogenic ADSCs (2.94, 95\% CI: $1.72 \sim 4.17)$ resulted in greater improvement than autologous or human ADSCs (0.90, 95\% CI: $0.26 \sim 1.54$; 1.21, 95\% CI: $0.81 \sim 1.61$, respectively). A high degree of heterogeneity existed in allogenic ADSCs $\left(I^{2}=84 \%\right)$. Based on the follow-up periods, ADSCs showed a similar effect on erectile function between follow-up periods of $<6$ weeks and $\geq 6$ weeks, and a high degree of heterogeneity remained. Substantial heterogeneity also existed in terms of both route of administration and whether co-intervention was applied or not (Table 3).

\section{Publication bias}

To interpret publication bias, contour enhanced funnel plot was applied. Conventional funnel plot is often interpreted as being caused by publication bias if asymmetry in the appearance of a funnel plot. On the other hand, contour enhanced funnel plot can be enhanced by adding contours of statistical significance to aid in interpreting the funnel plot. If studies appear to be missing in areas of low statistical significance, then it is possible that the asymmetry is due to publication bias. Publication bias was suspected when evaluated with a contour enhanced funnel plot (Fig. 3). We can re-display the funnel plot, taking into account the Trim and Fill adjustment. The trim and fill method is an iterative non-parametric technique to ad- just for publication bias by imputing studies estimated to be missing from the funnel plot asymmetry (37). The five imputed studies are shown as white circles, and the imputed estimates of the ICP/MAP ratios were reduced from 1.63 (95\% CI: $1.18 \sim 2.08)$ to 1.17 (95\% CI 0.64 1.70) after adjustment of the publication bias.

\section{Discussion}

Our meta-analysis included 19 studies that investigated the effects of ADSCs in rats with CNI-induced ED. The primary findings showed that ADSC therapy improved erectile function through increased ICP/MAP ratios. ADSC therapy also improved structural changes in the corpus cavernosume, which was evaluated by nNOS levels, cGMP, and CSM/collagen, suggesting that ADSCs had regenerated damaged cavernous tissues.

The nitric oxide (NO)-cGMP pathway is a main modulator in the corpus cavernosum for penile erection. NO produced by nNOS in nervous tissue leads to an increase in the intracellular cGMP concentration, which results in smooth muscle relaxation to attain a penile erection (38). During radical prostatectomy, the incision, heat, and mechanical stress may damage the cavernous nerve. CNI induces neurapraxia, decreased NOS, smooth muscle apoptosis, and penile fibrosis, which result in erectile dysfunction. Although the exact mechanism of ADSC therapy remains unclear, ADSCs have shown a paracrine

Table 3. Subgroup analysis to explore heterogeneity sources and changes in erectile dysfunction

\begin{tabular}{|c|c|c|c|}
\hline Subgroup & No. of trials $(\mathrm{Tn} / \mathrm{Cn})$ & SMD $(95 \% \mathrm{Cl})$ & $I^{2}$ \\
\hline \multicolumn{4}{|l|}{ Outcome assessment } \\
\hline Blinded & $5(64 / 64)$ & 1.33 (0.86 to 1.80$)$ & $23 \%$ \\
\hline Unblinded & $13(157 / 157)$ & 1.81 (1.17 to 2.46$)$ & $80 \%$ \\
\hline \multicolumn{4}{|l|}{ Cell origin } \\
\hline Autologous & $3(28 / 28)$ & 0.90 (0.26 to 1.54$)$ & $22 \%$ \\
\hline Allogenic & $8(79 / 79)$ & 2.94 (1.72 to 4.17$)$ & $84 \%$ \\
\hline Human & $7(114 / 114)$ & 1.21 (0.81 to 1.61$)$ & $41 \%$ \\
\hline \multicolumn{4}{|l|}{ Follow-up period } \\
\hline$<6$ weeks & $14(182 / 182)$ & 1.62 (1.12 to 2.12$)$ & $72 \%$ \\
\hline$\geq 6$ weeks & $4(39 / 39)$ & 1.73 (0.46 to 2.99$)$ & $80 \%$ \\
\hline \multicolumn{4}{|c|}{ Route of administration* } \\
\hline Intracavernous & $14(138 / 138)$ & 1.91 (1.26 to 2.56$)$ & $78 \%$ \\
\hline Cavernous nerve & $9(64 / 64)$ & 0.97 (0.43 to 1.50$)$ & $43 \%$ \\
\hline Others & $2(19 / 19)$ & 2.49 (0.30 to 4.69$)$ & $81 \%$ \\
\hline \multicolumn{4}{|l|}{ Co-intervention* } \\
\hline No & $17(149 / 149)$ & 1.67 (1.11 to 2.22$)$ & $72 \%$ \\
\hline Yes & $8(72 / 72)$ & 1.63 (1.18 to 2.08$)$ & $75 \%$ \\
\hline
\end{tabular}

Tn: numbers of treatment group, Cn: numbers of control group, SMD: standard mean difference, Cl: confidence interval.

*If studies evaluated multiple treatment groups in comparison to control groups, they were considered separate experiments. 

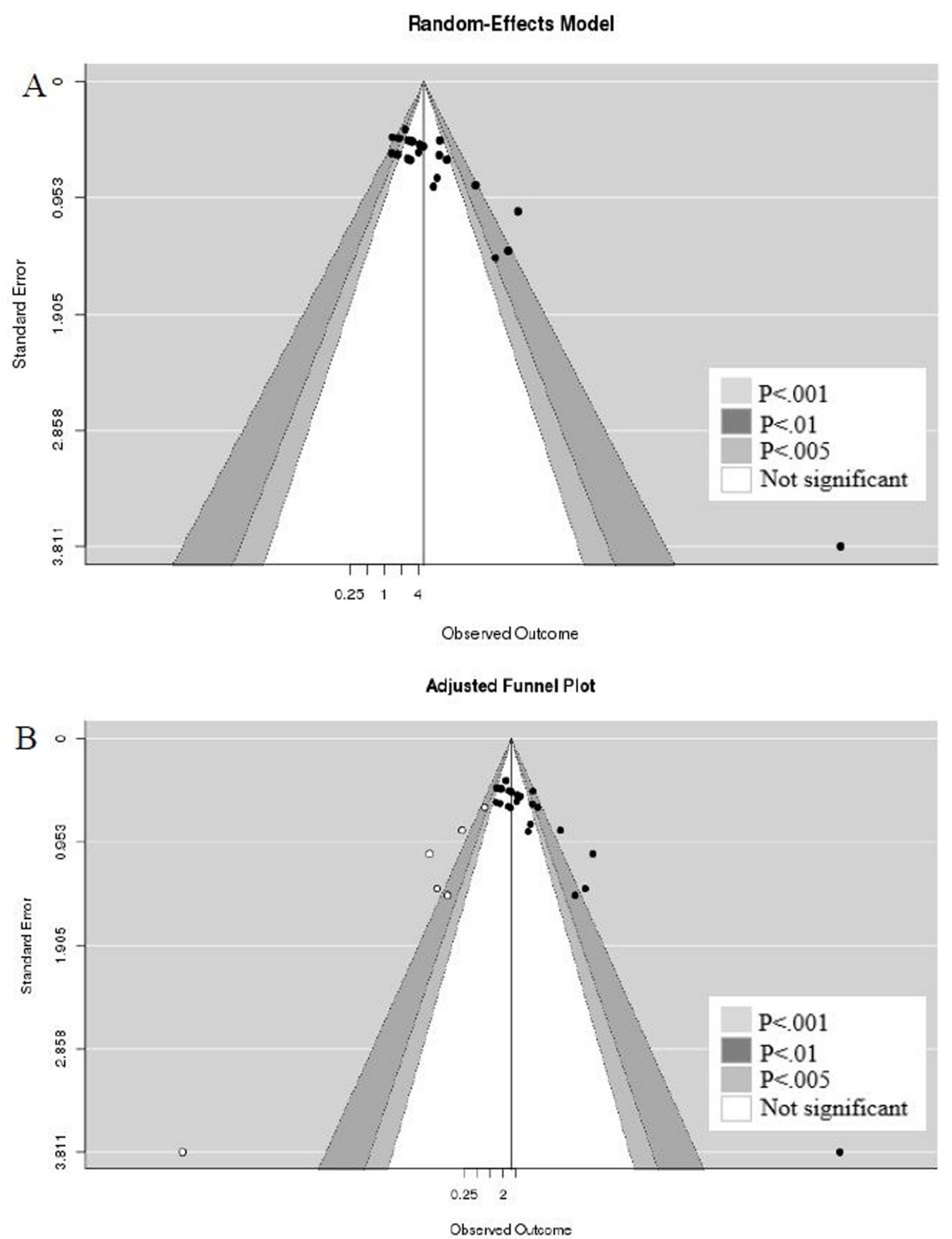

Fig. 3. Contour enhanced funnel plots displaying discrepancy between published data and after imputing studies estimated to be missing from the funnel plot asymmetry. Publication bias. (A) Unadjusted contour enhanced funnel plot and (B) adjusted contour enhanced funnel plot.

effect on surrounding smooth muscle, neurons, and endothelium to promote regeneration (10). To elucidate the mechanisms of ADSCs in improving erectile function, a study tracked ADSCs after injection in a CNI rat model using 5-ethynyl-2-deoxyuridine-labeled ADSCs. They had found that CNI upregulated stromal cell-derived factor-1 expression in the major pelvic ganglion (MPG), thereby attracting intracavernously injected ADSCs to the MPG to promote neuroregenerative effects on the cell bodies of injured nerves, resulting in enhanced erectile response (18).

There have been two previous meta-analysis studies on stem cells and ED. Hou et al. (13) had assessed 20 studies that reported that ADSCs significantly improved erectile function induced by various causes of ED in rat models.
The nNOS levels, cGMP, and CSM/collagen were also improved in the ADSC group when compared to the control. DM and CNI rats were the most commonly used rat models in the studies; however, the different ED models had no influence on the ADSC efficiency. Moderate to high heterogeneity $\left(I^{2}=68 \%\right)$ was suspected in the meta-analysis though. Shan et al. (14) had used data from 12 studies to analyze the effect of diverse sources of stem cells on CNI-induced ED. The meta-analysis showed a significant difference in erectile function as assessed by the ICP/MAP ratio between the stem cell and control groups (SMD $1.701,95 \%$ CI $1.245 \sim 2.158)$ with significant heterogeneity $\left(I^{2}=60.6 \%\right)$. The previous two studies had analyzed a variety of stem cells and ED models, but they did not evaluate 
the efficacy of stem cell treatments including the quality assessment of the studies.

To our knowledge, this is the first systematic review and meta-analysis to assess the effect of ADSCs on CNI-induced $\mathrm{ED}$. We evaluated more homogenous sources of stem cells and ED rat models. Moreover, we performed subgroup analysis to identify sources of heterogeneity including quality assessment. Randomization is important in clinical trials in order to balance the distribution of confounding factors. However, randomization in animal studies might have less of an effect on the results than in human clinical trials since animal studies utilize the same species with similar age and weight. On the other hand, blinding the outcome assessor might be crucial in animal studies because researchers may anticipate positive outcomes in their studies. When observer bias is introduced into intervention results, the efficacy of the intervention might be overestimated. In the current study, five of the 19 studies attempted to blind the outcome assessor $(20,21,23,24,35)$. Those studies showed a lesser effect on erectile function (1.33, 95\% CI: 0.86 to 1.80$)$ as compared to the non-blinded studies (1.81, 95\% CI: 1.17 to 2.46). In addition, heterogeneity was lower when the outcome assessment was blinded $\left(I^{2}=23 \%\right)$. Jeong et al. (39) had mentioned that the treatment effect may be overestimated when blinding was not maintained during the study. We assumed that the ADSC efficacy may be overestimated in studies a non-blinded outcome assessor. The cell origin subgroup showed that allogenic ADSCs were significantly more effective for erectile function than autologous or human ADSCs. None of the studies with allogenic ADSCs maintained blinding of outcome assessments.

This study had several limitations. The efficacy of ADSCs from this meta-analysis should be considered carefully because of the small number of studies. When outcome data were presented graphically in some studies, we extracted the numerical data from a graph using a program. Therefore, there might be discrepancies between the numerical data presented on the program and actual data. The quality assessment of studies was generally poor because the randomization method, allocation concealment, and blinding were not described. This poor methodological quality has weak internal validity. In addition, publication bias was also suspected. If the missing studies were systematically different, the results could be biased. Our results showed that the funnel plot was distributed asymmetrically around the mean effect size. Conradi et al. (40) had reported that published studies had more positive outcomes and statistically significant results than unpubli- shed studies in animal research.

\section{Conclusions}

Our meta-analysis indicates that ADSCs can be effective in improving erectile function and structural change of CNI-induced ED. However, caution should be used when evaluating the efficacy of ADSCs due to low methodological quality. A non-blinded outcome assessor may cause detection bias and overestimate ADSC efficacy. Therefore, the efficacy of ADSCs in animal models must be further evaluated with a rigorous study design in order to avoid bias.

\section{Acknowledgments}

This research was supported by the Bio \& Medical Technology Development Program of the National Research Foundation (NRF) funded by the Korean government (MSIT) (NRF-2017M3A9B8069577).

\section{Potential Conflict of Interest}

The authors have no conflicting financial interest.

\section{Author Contributions}

HJP participated in study concept and design, eligibility screening, data extraction, protocol preparation, data analysis, risk of bias assessment and writing the manuscript; JYL and HJ participated in study design, scientific concept, protocol preparation, eligibility screening, data extraction, data analysis and interpretation, risk of bias assessment and critical review of the manuscript; YHP participated in study design, data verification and critical review of the manuscript; HWY participated in study design, data analysis and interpretation and critical review of the manuscript; $\mathrm{UH}, \mathrm{SHH}$ and SWK participated in comment on protocol and critical review of the manuscript; NJK participated in literature search strategies, data search and validation. All authors read and approved the final manuscript.

\section{Supplementary Materials}

Supplementary data including two supplementary files can be found with this article online at http://pdf.medrang. co.kr/paper/pdf/IJSC/IJSC-12-s18122.pdf.

\section{References}

1. Bray F, Ferlay J, Soerjomataram I, Siegel RL, Torre LA, Jemal A. Global cancer statistics 2018: GLOBOCAN esti- 
mates of incidence and mortality worldwide for 36 cancers in 185 countries. CA Cancer J Clin 2018;68:394-424

2. Li J, Siegel DA, King JB. Stage-specific incidence rates and trends of prostate cancer by age, race, and ethnicity, United States, 2004-2014. Ann Epidemiol 2018;28:328-330

3. Mottet N, Bellmunt J, Bolla M, Briers E, Cumberbatch MG, De Santis M, Fossati N, Gross T, Henry AM, Joniau S, Lam TB, Mason MD, Matveev VB, Moldovan PC, van den Bergh RCN, Van den Broeck T, van der Poel HG, van der Kwast TH, Rouvière O, Schoots IG, Wiegel T, Cornford P. EAU-ESTRO-SIOG guidelines on prostate cancer. Part 1: screening, diagnosis, and local treatment with curative intent. Eur Urol 2017;71:618-629

4. Kang DH, Lee JY, Park SY, Moon HS, Jeong TY, Yoo TK, Choi HY, Park HY, Lee TY, Lee SW. Efficacy and safety of tadalafil $5 \mathrm{mg}$ administered once daily in Korean men with erectile dysfunction: a prospective, multicenter study. Korean J Urol 2010;51:647-652

5. Fode M, Ohl DA, Ralph D, Sønksen J. Penile rehabilitation after radical prostatectomy: what the evidence really says. BJU Int 2013;112:998-1008

6. Matz EL, Terlecki R, Zhang Y, Jackson J, Atala A. Stem cell therapy for erectile dysfunction. Sex Med Rev 2018 doi:10.1016/j.sxmr.2017.12.008

7. Gur S, Abdel-Mageed AB, Sikka SC, Hellstrom WJG. Advances in stem cell therapy for erectile dysfunction. Expert Opin Biol Ther 2018;18:1137-1150

8. Park JS, Suryaprakash S, Lao YH, Leong KW. Engineering mesenchymal stem cells for regenerative medicine and drug delivery. Methods 2015;84:3-16

9. Lin CS. Advances in stem cell therapy for the lower urinary tract. World J Stem Cells 2010;2:1-4

10. Peak TC, Anaissie J, Hellstrom WJ. Current perspectives on stem cell therapy for erectile dysfunction. Sex Med Rev 2016;4:247-256

11. Xin ZC, Xu YD, Lin G, Lue TF, Guo YL. Recruiting endogenous stem cells: a novel therapeutic approach for erectile dysfunction. Asian J Androl 2016;18:10-15

12. Dai R, Wang Z, Samanipour R, Koo KI, Kim K. Adipose-derived stem cells for tissue engineering and regenerative medicine applications. Stem Cells Int 2016;2016: 6737345

13. Hou QL, Ge MY, Zhang CD, Tian DD, Wang LK, Tian HZ, Wang WH, Zhang WD. Adipose tissue-derived stem cell therapy for erectile dysfunction in rats: a systematic review and meta-analysis. Int Urol Nephrol 2017;49:11271137

14. Shan H, Chen F, Zhang T, He S, Xu L, Wei A. Stem cell therapy for erectile dysfunction of cavernous nerve injury rats: a systematic review and meta-analysis. PLoS One 2015;10:e0121428

15. Muhlhausler BS, Bloomfield FH, Gillman MW. Whole animal experiments should be more like human randomized controlled trials. PLoS Biol 2013;11:e1001481

16. Hooijmans CR, Rovers MM, de Vries RB, Leenaars M, Ritskes-Hoitinga M, Langendam MW. SYRCLE's risk of bias tool for animal studies. BMC Med Res Methodol 2014; $14: 43$

17. Higgins JP, Thompson SG, Deeks JJ, Altman DG. Measuring inconsistency in meta-analyses. BMJ 2003;327: 557-560

18. Fandel TM, Albersen M, Lin G, Qiu X, Ning H, Banie L, Lue TF, Lin CS. Recruitment of intracavernously injected adipose-derived stem cells to the major pelvic ganglion improves erectile function in a rat model of cavernous nerve injury. Eur Urol 2012;61:201-210

19. Jeon SH, Shrestha KR, Kim RY, Jung AR, Park YH, Kwon O, Kim GE, Kim SH, Kim KH, Lee JY. Combination therapy using human adipose-derived stem cells on the cavernous nerve and low-energy shockwaves on the corpus cavernosum in a rat model of post-prostatectomy erectile dysfunction. Urology 2016;88:226.e221-e229

20. Jeong HH, Piao S, Ha JN, Kim IG, Oh SH, Lee JH, Cho HJ, Hong SH, Kim SW, Lee JY. Combined therapeutic effect of udenafil and adipose-derived stem cell (ADSC)/ brain-derived neurotrophic factor (BDNF)-membrane system in a rat model of cavernous nerve injury. Urology 2013; 81:1108.e1107-e1114

21. Kim IG, Piao S, Lee JY, Hong SH, Hwang TK, Kim SW, Kim CS, Ra JC, Noh I, Lee JY. Effect of an adipose-derived stem cell and nerve growth factor-incorporated hydrogel on recovery of erectile function in a rat model of cavernous nerve injury. Tissue Eng Part A 2013;19:14-23

22. Lee SH, Kim IG, Jung AR, Shrestha KR, Lee JH, Park KD, Chung BH, Kim SW, Kim KH, Lee JY. Combined effects of brain-derived neurotrophic factor immobilized poly-lactic-co-glycolic acid membrane with human adipose-derived stem cells and basic fibroblast growth factor hydrogel on recovery of erectile dysfunction. Tissue Eng Part A 2014;20: 2446-2454

23. Piao S, Kim IG, Lee JY, Hong SH, Kim SW, Hwang TK, Oh SH, Lee JH, Ra JC, Lee JY. Therapeutic effect of adipose-derived stem cells and BDNF-immobilized PLGA membrane in a rat model of cavernous nerve injury. J Sex Med 2012;9:1968-1979

24. You D, Jang MJ, Lee J, Suh N, Jeong IG, Sohn DW, Kim SW, Ahn TY, Kim CS. Comparative analysis of periprostatic implantation and intracavernosal injection of human adipose tissue-derived stem cells for erectile function recovery in a rat model of cavernous nerve injury. Prostate 2013; 73:278-286

25. Zheng T, Zhang TB, Wang CL, Zhang WX, Jia DH, Yang F, Sun YY, Ding XJ, Wang R. Icariside II promotes the differentiation of adipose tissue-derived stem cells to Schwann cells to preserve erectile function after cavernous nerve injury. Mol Cells 2018;41:553-561

26. Ying C, Yang $M$, Zheng X, Hu W, Wang X. Effects of intracavernous injection of adipose-derived stem cells on cavernous nerve regeneration in a rat model. Cell Mol Neurobiol 2013;33:233-240

27. Xu Y, Guan R, Lei H, Li H, Wang L, Gao Z, Song W, Xin $Z$. Therapeutic potential of adipose-derived stem 
cells-based micro-tissues in a rat model of postprostatectomy erectile dysfunction. J Sex Med 2014;11:2439-2448

28. Ying $\mathrm{C}, \mathrm{Hu} \mathrm{W}$, Cheng $\mathrm{B}$, Yang $\mathrm{M}$, Zheng $\mathrm{X}$, Wang $\mathrm{X}$. Erectile function restoration after repair of resected cavernous nerves by adipose-derived stem cells combined with autologous vein graft in rats. Cell Mol Neurobiol 2014;34: 393-402

29. Yang R, Fang F, Wang J, Guo H. Adipose-derived stem cells ameliorate erectile dysfunction after cavernous nerve cryoinjury. Andrology 2015;3:694-701

30. Chen X, Yang Q, Zheng T, Bian J, Sun X, Shi Y, Liang X, Gao G, Liu G, Deng C. Neurotrophic effect of adipose tissue-derived stem cells on erectile function recovery by pigment epithelium-derived factor secretion in a rat model of cavernous nerve injury. Stem Cells Int 2016;2016: 5161248

31. Lin H, Dhanani N, Tseng H, Souza GR, Wang G, Cao Y, Ko TC, Jiang H, Wang R. Nanoparticle improved stem cell therapy for erectile dysfunction in a rat model of cavernous nerve injury. J Urol 2016;195:788-795

32. Wu H, Tang WH, Zhao LM, Liu DF, Yang YZ, Zhang HT, Zhang Z, Hong K, Lin HC, Jiang H. Nanotechnology-assisted adipose-derived stem cell (ADSC) therapy for erectile dysfunction of cavernous nerve injury: in vivo cell tracking, optimized injection dosage, and functional evaluation. Asian J Androl 2018;20:442-447

33. Albersen M, Fandel TM, Lin G, Wang G, Banie L, Lin CS, Lue TF. Injections of adipose tissue-derived stem cells and stem cell lysate improve recovery of erectile function in a rat model of cavernous nerve injury. J Sex Med 2010;7: $3331-3340$
34. Lin G, Albersen M, Harraz AM, Fandel TM, Garcia M, McGrath MH, Konety BR, Lue TF, Lin CS. Cavernous nerve repair with allogenic adipose matrix and autologous adipose-derived stem cells. Urology 2011;77:1509.e1501el508

35. You D, Jang MJ, Kim BH, Song G, Lee C, Suh N, Jeong IG, Ahn TY, Kim CS. Comparative study of autologous stromal vascular fraction and adipose-derived stem cells for erectile function recovery in a rat model of cavernous nerve injury. Stem Cells Transl Med 2015;4:351-358

36. Bae JH, Shrestha KR, Park YH, Kim IG, Piao S, Jung AR, Jeon SH, Park KD, Lee JY. Comparison between subcutaneous injection of basic fibroblast growth factor-hydrogel and intracavernous injection of adipose-derived stem cells in a rat model of cavernous nerve injury. Urology 2014; 84:1248.e1241-e1247

37. Duval S, Tweedie R. Trim and fill: a simple funnelplot-based method of testing and adjusting for publication bias in meta-analysis. Biometrics 2000;56:455-463

38. Hallén K, Gustafsson LE, Wiklund NP. Nerve-induced release of nitric oxide from the rabbit corpus cavernosum is modulated by cyclic GMP. Faseb J 2005;19:A1205

39. Jeong H, Yim HW, Cho Y, Park HJ, Jeong S, Kim HB, Hong W, Kim H. The effect of rigorous study design in the research of autologous bone marrow-derived mononuclear cell transfer in patients with acute myocardial infarction. Stem Cell Res Ther 2013;4:82

40. Conradi U, Joffe AR. Publication bias in animal research presented at the 2008 Society of Critical Care Medicine Conference. BMC Res Notes 2017;10:262 